\title{
Induction of Preference and Performance after Acclimation to Novel Hosts in a Phytophagous Spider Mite: Adaptive Plasticity?
}

Anurag A. Agrawal, ${ }^{\star}$ Filipa Vala, and Maurice W. Sabelis

Section of Population Biology, University of Amsterdam, Kruislaan 320, Amsterdam 1098 SM, The Netherlands

Submitted November 9, 2000; Accepted October 29, 2001

ABSTRACT: We examined induction of preference and performance on novel host plants for two laboratory populations of the polyphagous spider mite Tetranychus urticae, with one population adapted to bean and the other population adapted to tomato. We bred four isofemale lines of the bean population only and used them in all the assays. The bean population had a $30 \%$ lower fecundity on tomato than on bean, while the tomato population had equal fecundity on both host plants. Acclimation of adult females to the novel host plant for both populations increased acceptability of that novel host but did not increase rejection of the original host. The bean population experienced a $60 \%$ benefit and a $30 \%$ cost in terms of egg production for acclimating to tomato, thus exemplifying adaptive plasticity. The tomato population showed a $23 \%$ benefit for acclimating to bean but no cost. Mites from the bean population that were acclimated to tomato fed more on tomato than did mites that were not acclimated to tomato. When these mites were fed inhibitors of cytochrome P-450 detoxification enzymes, their performance was severely depressed $(84 \%)$ on tomato but not on bean. However, mites that were fed inhibitors of P-450 enzymes did not reduce their acceptance of tomato as a host. Thus, performance on novel hosts (but not preference) in this species is likely correlated with the induction of detoxifying enzymes. Spider mites are known to form host races rapidly on novel hosts. Induction of preference and physiological acclimation via detoxification enzymes may enhance performance and, thus, strongly contribute to initial stages of host race formation.

Keywords: cytochrome P-450 enzymes, detoxification, diet specialization, host-range evolution, induction of preference, preferenceperformance relationships.

\footnotetext{
* Corresponding author. Address for correspondence: Department of Botany, University of Toronto, 25 Willcocks Street, Toronto, Ontario M5S 3B2, Canada; e-mail: agrawal@botany.utoronto.ca.
}

Am. Nat. 2002. Vol. 159, pp. 553-565. (C) 2002 by The University of Chicago. 0003-0147/2002/15905-0010\$15.00. All rights reserved.
Not all that is green is edible to herbivores. Herbivores have evolved mechanisms to circumvent or tolerate myriad plant defenses. Adaptations to plant defenses include behavioral tactics such as the removal of trichomes (Young and Moffett 1979; Tallamy 1986), the severance of defensedelivering canals (Dussourd 1993), or simply the switching of foods (Agrawal et al. 1999). In addition, herbivores employ enzymatic tactics to detoxify plant defensive compounds (Broadway 1995; Snyder and Glendinning 1996), they sequester defensive compounds (Bowers 1993), and they have compensatory feeding (Slansky and Feeny 1977). Such strategies promote the survival of herbivores and can be induced in response to consuming plant defenses (Snyder et al. 1993; Dussourd 1997; Agrawal 2001).

Induction of preference in insect herbivores is a classic area of study in plant-herbivore interactions that has not been integrated into the study of adaptation to plant defenses (reviewed by Papaj and Prokopy 1989; Szentesi and Jermy 1990; Bernays and Weis 1996). Typically, studies have shown that exposure of a herbivore to one food plant results in increased preference for that host in subsequent choice tests. Such studies, although great in number, usually lack information about the mechanisms of induced preference (i.e., is it habituation, physiological acclimation, imprinting, sensitization, increased acceptability, or associative learning [Bernays and Weiss 1996]) and the fitness consequences of induced preference (i.e., are there benefits and costs associated with the behavioral response [Karowe 1989]). To test whether induction of preference is an adaptive response to different plant defenses, we suggest that studies must incorporate measures of both preference and performance. Specifically, do herbivores prefer host plants on which they have previously fed because they have relatively high performance on those hosts?

Induction of preference for a novel host plant may result in higher performance on that host for several reasons. Most simply, if a host becomes more acceptable, the acclimated organism may feed more than if it is not acclimated. Other nonexclusive mechanisms include the in- 
duction of detoxification enzymes, which are known to play a role in metabolizing toxic compounds in many herbivores (Feyereisen 1999). Mixed-function oxidases are enzymes common to most arthropods and are thought to function in detoxification by catalyzing oxidative reactions (Mullin and Croft 1983; Strickler and Croft 1985; Brattsten 1988; Feyereisen 1999). In particular, cytochrome P-450 enzymes have been causally linked to detoxification of plant secondary metabolites in arthropods (Snyder and Glendinning 1996).

Local populations of herbivores may be specialized in host plant use (Fox and Morrow 1981; Thompson 1994), and this specialization may, in part, be a response to plant defenses. Variation among populations may be caused by genetic differentiation among populations, differences in induction of preference, and/or other environmental influences. If local specialization is genetically determined, then it can lead to genetic substructuring of populations, to formation of host races, and potentially to speciation as long as gene flow between populations is low (Prokopy et al. 1989; Via 1990; Mopper 1996). Induction of preference and performance is a mechanism that can lead to such genetic substructuring of populations because it increases host fidelity and decreases movement to alternative hosts (Via 1991). Phenotypic plasticity, in general, can promote local adaptation and potentially speciation by permitting flexible individuals initially to utilize and ultimately to adapt to marginal resources (West-Eberhart 1989). Substructuring caused by induction of preference, however, is subject to rapid breakdown caused by immigration or other factors that generate gene flow (Via 1991). The contribution of induction of host fidelity to restricted gene flow and genetic substructuring has been demonstrated in nature only for Rhagoletis flies feeding on hawthorn and apple fruits (Prokopy et al. 1982; Feder et al. 1994).

Induction of preference and performance may provide benefits similar to genetic specialization, such as a focused ability to find hosts and to deal with their defenses; induction is a more flexible strategy, however, that allows individuals to adapt to varying environments such as changes in host availability. Of course, genetic specialization and induction of preference and performance are not mutually exclusive (Prokopy et al. 1989; Feder et al. 1994). Within genetically differentiated host races of a species, induction of preference or performance may still partially explain host use. Genetic variation for preference and performance would indicate the potential for natural selection to act on host use, including phenotypically plastic traits such as induction of preference. Both theory and experiments have suggested that where selective agents are variable in space or time, phenotypic plasticity may evolve (Via and Lande 1985; Lively 1986; van Tienderen 1991;
Dudley and Schmitt 1996; Agrawal 1998). Adaptive phenotypic plasticity allows for the benefits of a particular phenotype to be experienced in an environment where they are needed, but the costs of expressing that phenotype are saved in environments where the trait is not needed. Induction of preference and performance is a form of phenotypic plasticity that may be particularly beneficial for generalist herbivores that use diverse host plants (i.e., allowing them to become temporary specialists). Thus, variable host plants may favor induction of preference and performance, and such induction may have evolutionary consequences for host-range use.

In this study we examine the influence of previous feeding experience on preference for and performance on novel host plants for two laboratory populations of spider mites, each one adapted to a different host plant. Specifically, we asked three questions. First, does acclimation to a novel host influence the acceptability of the novel host or the rejection of the original host? Second, is acclimation to a novel host beneficial (i.e., does it result in higher fecundity on the novel host) yet costly (i.e., does it result in lower fecundity on the original host)? Finally, are induction of preference and performance correlated with the amount of feeding on the novel host or the detoxification ability of the mites?

\section{Material and Methods}

\section{Study System and General Procedures}

The two-spotted spider mite Tetranychus urticae Koch, a generalist herbivore of worldwide importance as a crop pest (Helle and Sabelis 1985), has been recorded from more than 900 host-plant species (Bolland et al. 1998). Tetranychus urticae feed by piercing leaf parenchyma cells with their stylets and sucking out the cell contents. If a host plant is unsuitable or has been overexploited, mites will disperse passively as aerial plankton (Kennedy and Smitley 1985). Mites typically disperse as adult females. Once they are on a new host, they can accept that host or reject it by walking onto touching hosts or again dispersing by air (Byrne et al. 1982; Kennedy and Smitley 1985).

Evidence suggests that, under many circumstances, local populations of T. urticae form host races that are genetically distinct from other populations (Gould 1979; Fry 1989; Gotoh et al. 1993; Tsagkarakou et al. 1997; Agrawal $2000)$. The fast generation time $\left(10-12 \mathrm{~d}\right.$ at $\left.25^{\circ} \mathrm{C}\right)$ and relatively low mobility of spider mites, coupled with the heterogeneous environment (i.e., various host plants), are thought to contribute to localized host races. Host race formation is often associated with adaptation to the toxic properties of particular host-plant species, and spider 
mites remain one of the classic systems in which hostrange evolution appears to be constrained by trade-offs in fitness on alternative hosts (Gould 1979; Fry 1990; Agrawal 2000; Yano et al. 2001).

In this study, two laboratory populations of T. urticae were used, one that is adapted to feeding on bean (henceforth, the B-population) and one that is adapted to feeding on tomato (henceforth, the T-population). Common bean (Phaseolus vulgaris var. Arena) is a highly suitable host for T. urticae, and the B-population has been continuously maintained in cultures of detached bean leaves placed on wet cotton wool for at least 8 yr. Four isofemale lines were developed from the B-population. Because T. urticae is a haplodiploid species in which fertilized eggs develop into females and unfertilized eggs develop into males, isofemale lines can be created by mother-son matings. From a large number of B-population mites ( $\gg 500$ individuals), four individual unmated females were isolated and mated with their haploid sons. We repeated this procedure for four consecutive generations, and henceforth, each isofemale line was maintained on individual cultures of detached bean leaves. Individuals within such an isofemale line are expected to have an inbreeding coefficient of 0.9 and, thus, can be effectively thought of as near-clones. Since each of the isofemale lines is extremely inbred, we assume that inbreeding depression effects are consistent across the four populations. In all experiments with the B-population, we used equal numbers from each of the four isofemale lines and included this term as a random effect in the statistical models (see below).

Tomato is typically a hostile host for T. urticae because of phytochemicals in the leaves and glandular trichomes (Aina et al. 1972; Chatzivasileiadis and Sabelis 1997). However, T. urticae can adapt to tomato plants (i.e., evolve higher fitness on tomato) within 10 generations (Fry 1990). The T-population we used has been maintained in a culture of at least several hundred individuals on intact tomato plants (Lycopersicon esculentum var. Moneymaker) for 3 yr. Isofemale lines were not established for the Tpopulation because of time constraints.

All experiments were conducted in a growth room on a $16 \mathrm{~L}: 8 \mathrm{D}$ cycle at $25^{\circ} \mathrm{C}$ constant temperature and $50 \%$ relative humidity.

\section{Performance of the Two Laboratory Populations of Mites}

Survival of the adult females and fecundity (number of eggs laid) over $5 \mathrm{~d}$ was assayed for each of the B- and Tpopulation mites on both bean and tomato. Inseminated adult females were individually placed on $1.5-\mathrm{cm}$-diameter leaf disks of bean or tomato interdigitated, but not touching, on wet cotton wool in plastic trays ( 80 disks per tray). This arrangement keeps the leaf disks turgid and provides a barrier (wet cotton) to prevent the mites from traveling between leaf disks. As in all experiments, replicates of Bpopulation mites were equally subdivided into the four isofemale lines ( $n \approx 12$ mites for each line, each treatment, and each trial for two trials). Trials were temporally separated by at least $1 \mathrm{wk}$ and were conducted with separate sets of mites but were otherwise identical. Fecundity of Bpopulation mites was analyzed with a fully factorial ANOVA, with host plant, isofemale line, and trial as factors. Fecundity of T-population mites on the two hosts was analyzed with a $t$-test $(n=56$ for each treatment for one trial). In this and all subsequent experiments, fecundity is only reported for mites that survive. Mortality in each population was analyzed with a $G$-test for a $2 \times 2$ frequency table, with host plant (bean or tomato) and status of the mites (alive or dead) as factors.

\section{Mixed-Model Analyses}

In the above and all subsequent mixed-model analyses, host plant was treated as a fixed effect, and isofemale line and trial were treated as random effects. By treating isofemale line as a random effect, we gain the ability to generalize significant effects of the isofemale line (and interactions) as significant genetic variation for the traits (Zar 1996). Since all experiments were conducted in the laboratory, trial was treated as an arbitrary blocking factor included in the analyses to correct for variance associated with the different trials. Thus, trial by treatment interaction was not included in the model (model 2 described by Newman et al. 1997). Mixed-model analyses were conducted with SAS PROC MIXED (SAS Institute 1999). Degrees of freedom for $F$-tests of fixed effects were estimated using the Satterthwaite approximation (SAS Institute 1999). As suggested by the SAS Institute (1999), the likelihood-ratio $\chi^{2}$ test was employed for tests of the random effects. The likelihood-ratio $\chi^{2}$ test is a one-sided single degree of freedom test that tests the hypothesis that the variation caused by the random effect is greater than zero.

\section{Induced Acceptability of a Novel Host}

In this experiment with B- and T-population mites, we asked whether acclimation to a novel host affected the acceptability of the novel host. Because passively dispersing adult mites do not typically encounter a choice between hosts, we examined the acceptability of hosts following acclimation to either bean or tomato plants. We believe that acceptability of hosts is a biologically more relevant measure of preference for mites than strict choice. Acceptance of a host was defined as a mite staying on the host on which it was placed; alternatively, the mite could reject the host and move to another leaf disk (of the other plant 
species) that was touching the disk to which the mite was introduced. The relatively low mobility of mites makes our measure of rejection realistic because mites must actively move to get to the other host.

Inseminated adult female mites were taken from the bulk cultures and individually acclimated to bean or tomato for $5 \mathrm{~d}$. We interdigitated 1.5-cm-diameter leaf disks of bean and tomato on wet cotton wool in plastic trays (80 disks per tray) and transferred a single mite to each disk. For each of the two populations, 160 mites were divided into two groups, one acclimated to bean and the other to tomato. After $5 \mathrm{~d}$ of feeding, each mite was subjected to one of two treatments: introduction to a tomato leaf disk that was touching a bean leaf disk or introduction to a bean leaf disk that was touching a tomato leaf disk. After accounting for the mortality in the $5 \mathrm{~d}$ of acclimation, we established a total of 296 such arenas, with each population having individuals acclimated to bean or tomato being placed on bean or tomato leaf disks. We assayed acceptability of the host to which the mites were introduced by examining the location of the mites $1.5 \mathrm{~h}, 6 \mathrm{~h}$, and $3 \mathrm{~d}$ after the transfer. Although there was some mortality when acclimating the isofemale lines, we assume that genetic variation within isofemale lines is too small to be relevant for selection to alter the outcome (as an alternative to induction). Although mortality was relatively low in the T-population, selection cannot be excluded from contributing to the effects observed.

We statistically analyzed acceptability of a single host within each population with a $G$-test for a $2 \times 2$ frequency table, with acclimation host and mite location as factors. Four such analyses were conducted because the acceptability of tomato and bean was independently analyzed for the B-population and T-population. We performed G-tests for each of the three sampling times and adjusted probabilities using a sequential Bonferroni correction (Rice 1989).

\section{Induced Performance on a Novel Host}

In this experiment, we examined the fecundity of mites in both populations after acclimation to the two hosts and transfer to a new host. The design was identical to that of the previous experiment except that, after acclimation for $5 \mathrm{~d}$, mites were placed onto single isolated leaf disks (bean or tomato). This experiment was replicated twice with each mite population, and sample sizes were $\sim 20$ mites per treatment combination (two acclimation hosts $\times$ two assay hosts $\times$ two trials). The number of eggs laid $2 \mathrm{~d}$ and $4 \mathrm{~d}$ following the transfer was counted. We analyzed fecundity of B-population mites with SAS PROC MIXED, with acclimation plant (fixed effect), assay plant (fixed effect), isofemale line (random effect), and trial (random effect) as factors. We conducted separate analyses for the number of eggs laid on days 1 and 2 and days 3 and 4 . For the T-population, analyses were identical except for the exclusion of the isofemale line term.

\section{Mechanisms of Induced Performance}

Test for Effects on Amount of Feeding. In order to determine whether acclimated mites feed more than control mites, we conducted an experiment with 160 mites from the Bpopulation divided into four groups in a $2 \times 2$ factorial design similar to that outlined above (i.e., acclimated to bean or tomato and assayed on bean or tomato). After the mites were individually acclimated for $5 \mathrm{~d}$ and transferred to the second host, they were repeatedly monitored for feeding. Each mite was observed four times beginning $2 \mathrm{~h}$ after the transfer and continuing every $2 \mathrm{~h}$ thereafter. Feeding was determined under a dissecting microscope in a very bright room with no additional lighting (i.e., no light that could have disrupted their normal behavior was focused on the mites). Three factors were used to determine whether the mite was feeding: insertion of the chelicerae in the leaf tissue; visual movement of fluids inside the transparent mite; and a slow, rhythmic movement of the mouthparts. We analyzed data with $G$-tests on the frequency of mites feeding. For each sample, we carried out two $G$-tests, one for comparing bean- and tomatoacclimated mites transferred to tomato and one for comparing bean- and tomato-acclimated mites transferred to bean. Each of these tests addresses whether acclimation affects feeding on the assay host. Alpha values were adjusted using the sequential Bonferroni correction for the four repeated comparisons (Rice 1989).

Test for Effects on Consumption. We conducted an additional experiment in which we measured the amount of leaf tissue consumed on the novel host following acclimation. This allowed us to use leaf tissue consumed as a covariate in the statistical analysis to examine whether the effects of acclimation could be explained by changes in consumption. In other words, does acclimation involve more than increased feeding? We tested only the Bpopulation mites. Mites were individually acclimated to bean or tomato and then transferred to tomato $(n=10$ mites per isofemale line per treatment, for a total of 80 mites). We recorded the number of feeding scars $(\approx 0.1$ $\left.\mathrm{mm}^{2}\right) 6 \mathrm{~h}$ after the transfer. We analyzed the effects of isofemale line and acclimation host on the number of feeding scars after $6 \mathrm{~h}$ with a two-factor analysis in SAS PROC MIXED. We also recorded the number of scars and eggs laid after $2 \mathrm{~d}$ for the tomato leaf disks only. We analyzed the effects of isofemale line and acclimation host with a three-factor analysis, with the number of feeding 


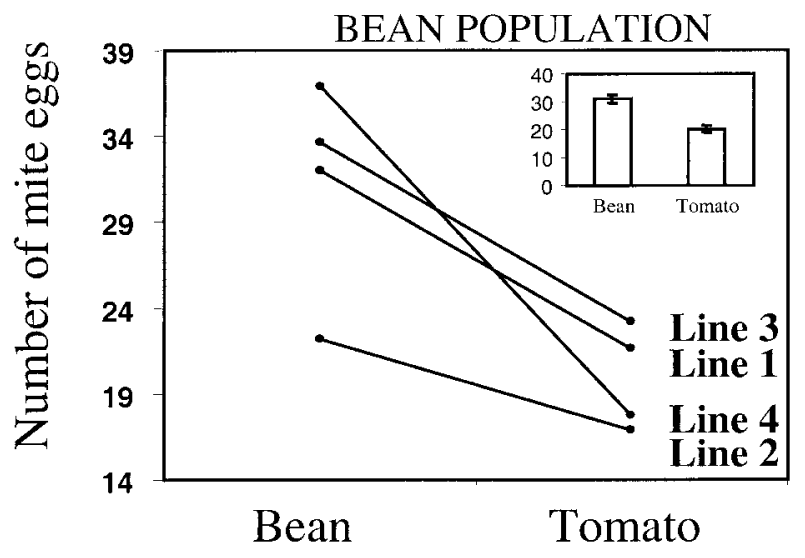

Assay host

Figure 1: Norm of reaction plot for the effect of two host plants, bean and tomato, on the number of eggs laid over $5 \mathrm{~d}$ by four isofemale lines of Tetranychus urticae raised on bean. Inset bar graph shows the mean \pm SE eggs laid across the four isofemale lines.

scars as a covariate. A significant effect of acclimation host in this model (irrespective of number of feeding scars) would indicate that effects of acclimation on egg production involves more than an increased amount of feeding (i.e., a higher conversion efficiency, sensu Karowe 1989).

Test for Role of P-450 Detoxification Enzymes. To examine the role of P-450 enzymes in the acclimation of the Bpopulation to tomato plants, we conducted an experiment using inhibitors of P-450 enzymes. Piperonyl butoxid (2(2-Butoxyethoxy)ethyl-6-propylpiperonylether; PB) has long been known to inhibit P-450 enzymes in arthropods (Cassida 1970) and has recently been used to establish a causal connection between P-450 enzymes and detoxification of plant allelochemicals (Snyder and Glendinning 1996). In T. urticae, PB inhibits aldrin epoxidase (Mullin et al. 1984). We dissolved PB (Sigma Chemical, St. Louis) in acetone and then diluted the solution with an equal

Table 1: Mixed-model analysis for effects of host plant, mite isofemale line, and trial on reproduction of bean-adapted mites (B-population) using SAS PROC MIXED

\begin{tabular}{lrrrr}
\hline Source & df & $F$ & \multicolumn{1}{c}{$\chi^{2}$} & \multicolumn{1}{c}{$P$} \\
\hline Host plant $(H)$ & 1,3 & 23.36 & & $\mathbf{. 0 1 7}$ \\
Mite line $(M)^{\mathrm{a}}$ & 1 & & 1.2 & .137 \\
$H \times M^{\mathrm{a}}$ & 1 & & 1.6 & .103 \\
Trial $^{\mathrm{a}}$ & 1 & & 41.7 & $<.001$ \\
\hline
\end{tabular}

Note: Boldface type indicates $P<.05$.

a Likelihood-ratio $\chi^{2}$ test for random effects.

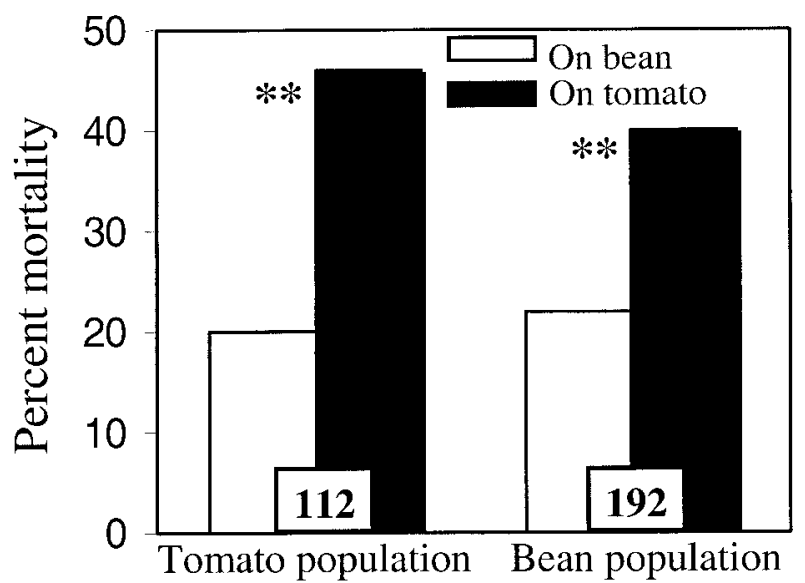

Figure 2: Percent mortality of adults of T- and B-population mites on two host plants, bean and tomato. Two asterisks indicate significant differences at $P<.05$ using a $G$-test. Sample sizes are given at the base of the bars.

part of water, resulting in 2,000 parts per million PB by volume, which is a nontoxic dose of PB (see details in Strickler and Croft 1985). Approximately $50 \mu \mathrm{L}$ of the $\mathrm{PB}$ solution was painted on each treatment leaf disk, and 50 $\mu \mathrm{L}$ of $1: 1$ acetone : water solution was painted on control leaf disks. We examined the B-population mites using three pairs of comparisons: control and PB-treated bean leaves ( $n=20$ for each treatment), control and PB-treated tomato leaves ( $n=20$ for each treatment), and mites acclimated to tomato on control and PB-treated tomato ( $n=40$ for each treatment). We assayed mites for egg production after $3 \mathrm{~d}$. The first comparison was used to verify that painting leaves with $\mathrm{PB}$ would have minimal negative effects on mites on a suitable host plant that probably does not require P-450 mediated detoxification (Mullin and Croft 1983). The second and third comparisons were used to examine the effects of P-450 enzymes on detoxification of tomato, the host that putatively requires detoxification. For the three comparisons, we analyzed the effects of $\mathrm{PB}$ treatment and isofemale line on egg production with SAS PROC MIXED.

To examine further the role of P-450 enzymes in the acclimation of the B-population to tomato plants, we conducted an "acceptability" assay. Mites were acclimated to tomato for $5 \mathrm{~d}$ and then were subject to two different treatments for $3 \mathrm{~d}$ : they were either transferred to tomato treated with PB (as above; $n=29$ mites) or transferred to tomato treated with the control acetone : water solution ( $n=35$ mites). After these treatments were imposed, we placed each mite on a fresh tomato leaf disk that was touching a bean leaf disk. We hypothesized that inhibition of the P-450 enzymes may reduce the acceptability of to- 
$\square$ Acclimated on bean

Acclimated on tomato
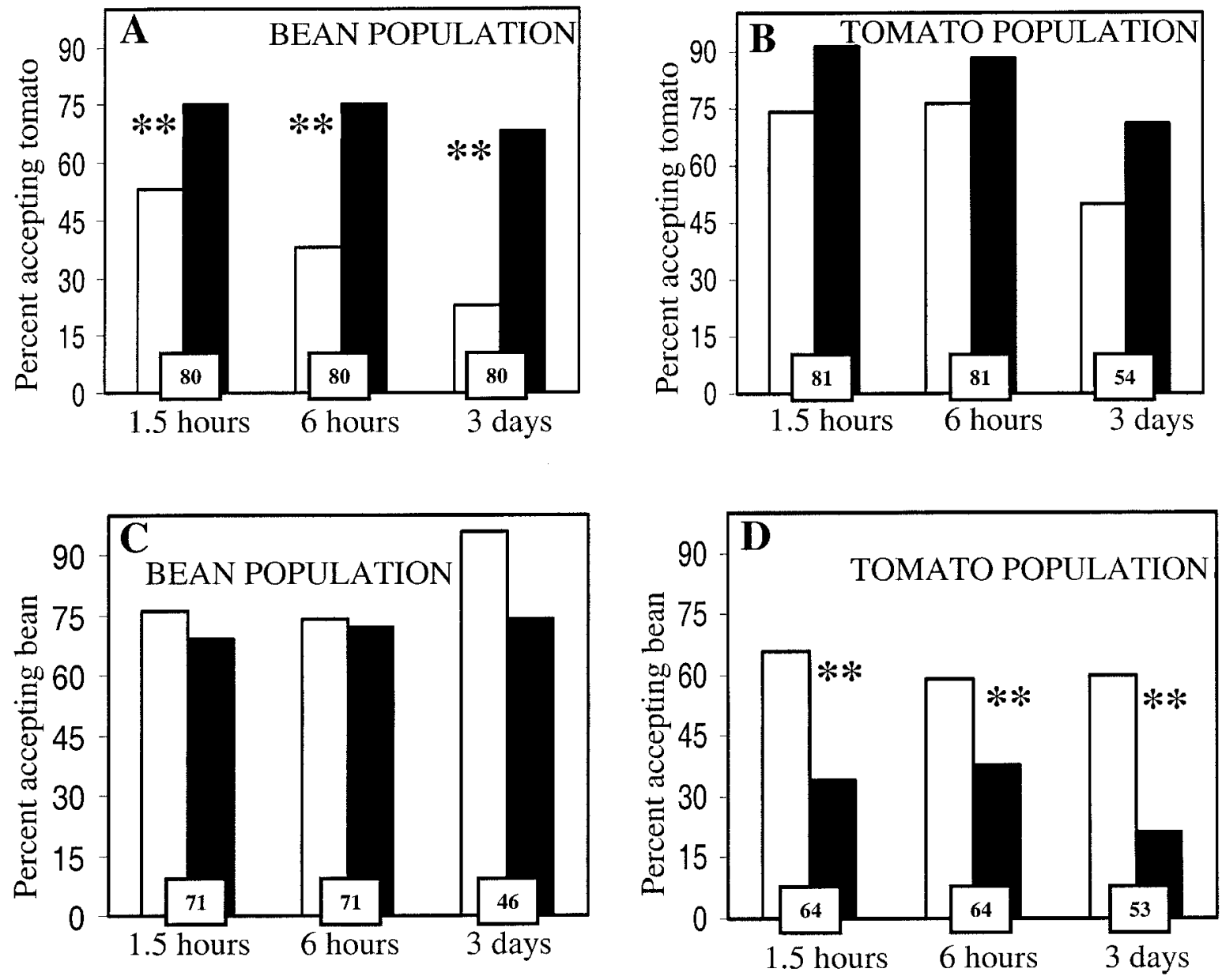

Figure 3: Induced acceptability of either tomato or bean after acclimation to tomato or bean. $A$, $C$, Results with B-population mites. $B$, $D$, Results with T-population mites. Note that $A$ and $B$ show acceptance of tomato (i.e., the mite was placed on a tomato leaf next to a bean leaf) and $C$ and $D$ show acceptance of bean. Two asterisks indicate significant differences at $P<.05$ using a $G$-test after sequential Bonferroni correction. Sample sizes are given at the base of the bars.

mato because the enzymes are needed for detoxification. The effect of PB-mediated P-450 enzyme inhibition on the acceptability of tomato was assayed after $3 \mathrm{~h}$ and again after $3 \mathrm{~d}$. We used $G$-tests to analyze the data, and we adjusted $\alpha$ values using the sequential Bonferroni correction for the two repeated comparisons (Rice 1989).

\section{Results}

Performance of the Two Populations of Mites

Fecundity of B-population mites was 30\% lower on tomato than on bean (fig. 1; table 1), and the four iso- female lines did not differ in the extent to which tomato was a poor host (no host plant $\times$ mite population interaction in table 1). Fecundity of T-population mites was equal on tomato and bean (mean $\pm \mathrm{SE}$; tomato $=33.480 \pm 2.176$, bean $=33.565 \pm 0.945$; separate variance $t$-test, $\mathrm{df}=32.6, t=0.036, P=.972$ ). Both populations of mites had higher mortality on tomato ( $>40 \%)$ than on bean $(<20 \%$; fig. 2$)$.

\section{Induced Acceptability of a Novel Host}

Acclimation to a novel host plant increased acceptability of that novel host (fig. $3 A, 3 D$ ) but did not increase re- 

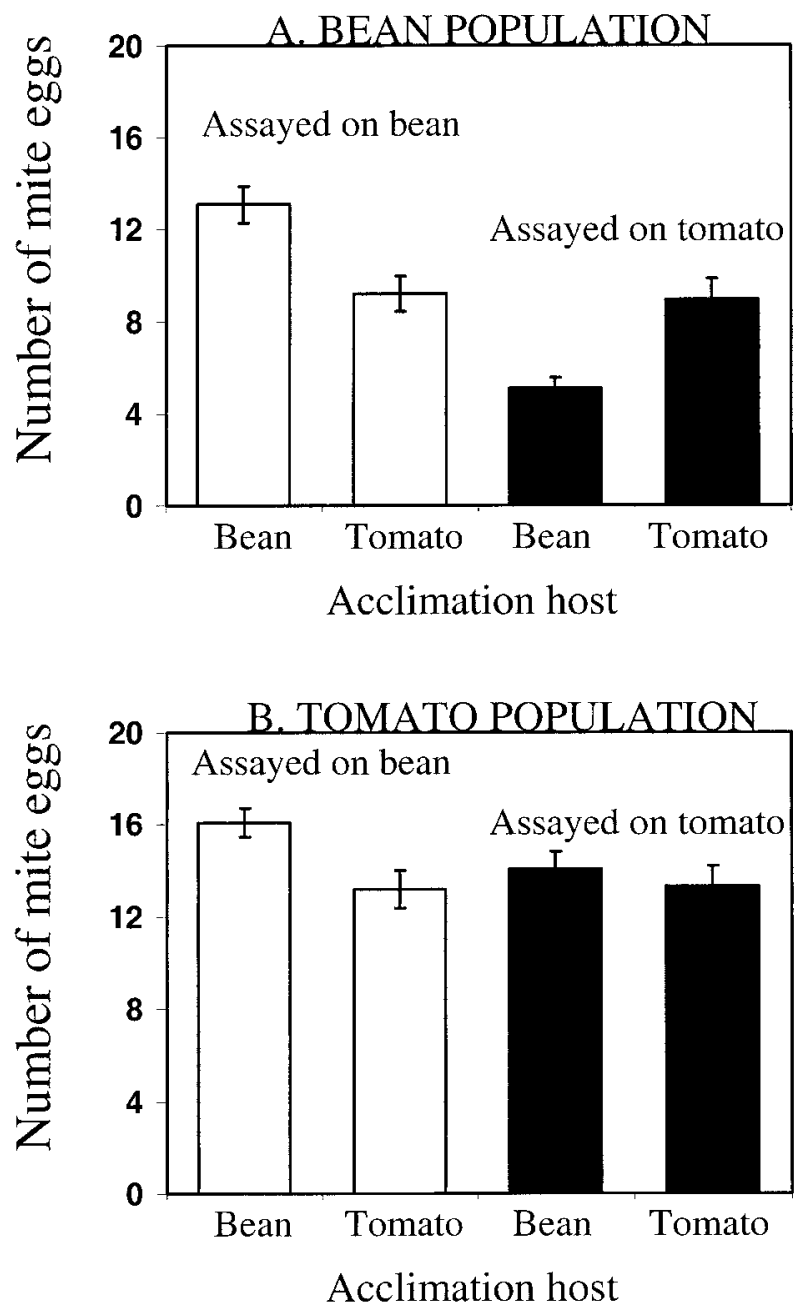

Figure 4: Two-day fecundity of B- and T-population mites after acclimation to either bean or tomato. Bars are mean \pm SE.

jection of the original host (fig. 3B, 3C). For B-population mites, acceptability of the novel host (tomato) was more than three times more likely for mites acclimated to tomato after $3 \mathrm{~d}$ compared with mites acclimated to bean. In the T-population, acceptance of the novel host (bean) was also three times more likely for mites acclimated to bean compared with mites acclimated to tomato. Acclimation to bean or tomato did not affect the acceptability of the original host in both populations of mites. These effects were consistent across the three time periods in which the mites were assayed (fig. 3).

We further analyzed the data for genetic variation in induced acceptance of the B-population mites. Across the two trials, approximately $80 \%$ of mites in isofemale lines 1,2 , and 4 were found on the host to which they were acclimated (i.e., stayed on the acclimation host or moved from the other host to the acclimation host) by day 3 , whereas only $50 \%$ of mites from isofemale line 3 were found on the host to which they were acclimated ( $G=$ 10.501, $P=.015$ ).

In these trials, mortality during the acclimation period was quite low on tomato (8\%; fig. 3) compared with that during the performance trials (fig. 2). In addition, an unexplained mortality pattern emerged within the 3-d assay period (fig. 3): mortality was high when mites were assayed for acceptance on their home host (i.e., B-line on bean) but quite low when mites were assayed on the foreign host.

\section{Induced Performance on a Novel Host}

The B-population mites performed best when acclimated to and assayed on bean and worst when acclimated to bean and assayed on tomato (fig. $4 A$; table $2 A$ ). Mites acclimated to tomato performed equally well on bean and tomato. The acclimation host interacted with the assay host to affect mite fecundity (table $2 A$ ). The $30 \%$ lower fecundity of mites acclimated to tomato and assayed on bean compared with mites acclimated to and assayed on bean can be interpreted as a phenotypic cost of acclima-

Table 2: Mixed-model analysis for effects of acclimation host plant, assay host plant, mite isofemale line, and trial on reproduction of bean-adapted mites (B-population) using SAS PROC MIXED

\begin{tabular}{lllll}
\hline Source & $\mathrm{df}$ & $F$ & $\chi^{2}$ & $P$ \\
\hline
\end{tabular}

\begin{tabular}{lrrrr}
\hline $\begin{array}{l}\text { A. Reproduction for days } 1 \text { and } 2 \\
\quad \text { following transfer to }\end{array}$ & & & & \\
$\quad$ assay host plant: & & & & \\
Acclimation host $(H)$ & 1,3 & .04 & .856 \\
Assay host $(A)$ & 1,3 & 10.59 & & $\mathbf{. 0 4 7}$ \\
$H \times A$ & 1,3 & 25.11 & & $\mathbf{. 0 1 5}$ \\
Mite line $(M)^{\mathrm{a}}$ & 1 & & .4 & .264 \\
Trial & 1 & & 0 & .500 \\
$H \times M^{\mathrm{a}}$ & 1 & & 0 & .500 \\
$A \times M^{\mathrm{a}}$ & 1 & & 1.4 & .118 \\
$H \times A \times M^{\mathrm{a}}$ & 1 & & 0 & .500
\end{tabular}

B. Reproduction for days 3 and 4

following transfer to

assay host plant:

Acclimation host

Assay host $(A)$

$H \times A$

Mite line $(M)^{\mathrm{a}}$

Trial $^{\mathrm{a}}$

$H \times M^{\mathrm{a}}$

$A \times M^{\mathrm{a}}$

$H \times A \times M^{\mathrm{a}}$

\begin{tabular}{rrrr}
1,3 & 1.81 & & \\
1,3 & 3.62 & & .153 \\
1,3 & .15 & & .724 \\
1 & & .4 & .264 \\
1 & & 0 & .500 \\
1 & & 0 & .500 \\
1 & & 3.3 & .035 \\
1 & & 0 & .500 \\
\hline
\end{tabular}

Note: Boldface type indicates $P<.05$.

${ }^{a}$ Likelihood-ratio $\chi^{2}$ test for random effects. 
Table 3: Mixed-model analysis for effects of acclimation host plant, assay host plant, and trial on reproduction of tomatoadapted mites (T-population) using SAS PROC MIXED

\begin{tabular}{|c|c|c|c|c|}
\hline Source & $\mathrm{df}$ & $F$ & $\chi^{2}$ & $P$ \\
\hline \multicolumn{5}{|c|}{$\begin{array}{l}\text { A. Reproduction for days } 1 \text { and } 2 \\
\text { following transfer to } \\
\text { assay host plant: }\end{array}$} \\
\hline Acclimation host $(H)$ & 1,126 & 8.19 & & .005 \\
\hline Assay host $(A)$ & 1,126 & 1.72 & & .192 \\
\hline$H \times A$ & 1,126 & 1.78 & & .184 \\
\hline Trial $^{\mathrm{a}}$ & 1 & & 138.8 & $<.001$ \\
\hline \multicolumn{5}{|c|}{$\begin{array}{l}\text { B. Reproduction for days } 3 \text { and } 4 \\
\text { following transfer to } \\
\text { assay host plant: }\end{array}$} \\
\hline Acclimation host $(H)$ & 1,107 & 3.22 & & .076 \\
\hline Assay host $(A)$ & 1,107 & 0 & & .960 \\
\hline$H \times A$ & 1,107 & 0 & & .988 \\
\hline Trial $^{\mathrm{a}}$ & 1 & & 2.6 & .53 \\
\hline
\end{tabular}

Note: Boldface type indicates $P<.05$.

${ }^{\text {a }}$ Likelihood-ratio $\chi^{2}$ test for random effects.

tion. The $60 \%$ higher fecundity of mites acclimated to and assayed on tomato compared with mites acclimated to bean and assayed on tomato is the short-term fitness benefit of acclimation. As expected, the effects of acclimation were only detectable on the first $2 \mathrm{~d}$ of the assay and were no longer detectable on days 3 and 4 (acclimation host $x$ assay host interaction is no longer significant in table $2 B$ ). This result indicates that $2 \mathrm{~d}$ is sufficient for acclimation of the mites to novel hosts because the unacclimated mites performed as well as the acclimated mites on days 3 and 4.

The T-population mites perform best when acclimated to bean (fig. $4 B$; table $3 A$ ). The positive effect of acclimation to bean $(\approx 15 \%$ more eggs overall), irrespective of the assay host, was seen in the analysis of eggs produced in the first $2 \mathrm{~d}$ after transfer and marginally for the eggs produced on days 3 and 4 after the transfer (fig. $4 B$; table 3). However, we detected no statistical interaction between the acclimation host and assay host for effects on mite reproduction, thereby finding no evidence for an effect of acclimation on performance. In a post hoc analysis, we examined the effect of acclimation to bean and tomato on performance on the two assay hosts independently. In this analysis of the T-population, mites acclimated to bean had a $23 \%$ higher fecundity on bean than mites acclimated to tomato $(F=9.93, \mathrm{df}=1,63, P=.003)$, indicating a benefit of acclimation to the novel host, bean. Conversely, we detected no effect of acclimation host on mite performance on tomato $(F=1.02$, $\mathrm{df}=1,62, P=.317)$, indicating no cost of acclimation. In this post hoc analysis, even if we accept a corrected $\alpha=0.025$ for rejection of the null hypothesis, we still detect a robust benefit of acclimation to bean on performance.

\section{Mechanisms of Induced Performance}

To test whether the effects of acclimation on mite performance were caused in part by increased feeding, we examined feeding behavior of acclimated and unacclimated mites on both hosts using the B-population. After sequential Bonferroni correction, none of the differences was significant (table 4). Nevertheless, in an overall analysis in which we summed all feeding observations, $88 \%$ of mites acclimated to tomato and assayed on tomato were found to be feeding at least once, whereas only $65 \%$ of mites acclimated to bean and assayed on tomato were found to be feeding ( $P=.01$; table 4$)$. In the overall analysis, no difference in feeding was observed for the mites assayed on bean. Thus, the benefit of acclimation to tomato may be affected by the amount of feeding, but the cost of acclimation to tomato does not appear to involve effects on feeding.

In the acclimation experiment in which we measured feeding scars after $6 \mathrm{~h}$, mites acclimated to tomato were more likely to feed on tomato; this effect was marginally variable among isofemale lines (effect of acclimation host, $F=20.27, \mathrm{df}=1,3, P=.021$; isofemale line, $\mathrm{df}=1$, $\chi^{2}=0, \quad P=.500 ;$ acclimation host $\times$ isofemale line, df $=1, \chi^{2}=2.2, P=.069$; data not shown). After $2 \mathrm{~d}$, effects of acclimation on egg production on tomato were present (mean \pm SE eggs; acclimation host, bean = $5.152 \pm 0.628$, tomato $=7.789 \pm 0.776$ ); however, they were almost entirely explained by the number of feeding scars (table 5). When the number of feeding scars was pooled across acclimation treatment and isofemale lines, we found a very strong association with the number of eggs produced (linear regression, $r^{2}=0.806, \quad F=$ 287.056, $\mathrm{df}=1,69, P<.001$; fig. 5). Thus, the positive effects of acclimation are caused by the increased amount of feeding, not increased efficiency in converting ingested tissue to eggs.

Piperonyl butoxide, an inhibitor of P-450 enzymes in arthropods, did not decrease performance of the B-population mites when assayed on bean (treatment, $F=$ $0.54, \mathrm{df}=1,3, P=.517$; isofemale line, $\mathrm{df}=1, \chi^{2}=$ $0, P=.500$; treatment $\times$ isofemale line, $\mathrm{df}=1, \chi^{2}=$ $1.2, P=.137$; fig. 6 ). Nevertheless, there was an $84 \%$ reduction in the number of eggs produced by enzymeinhibited mites on tomato compared with controls (treatment, $F=27.57, \mathrm{df}=1,3, P=.014 ;$ isofemale line, $\mathrm{df}=1, \chi^{2}=0.6, P=.219$; treatment $\times$ isofemale line, $\mathrm{df}=1, \chi^{2}=0, P=.500$; fig. 6). When we acclimated B-population mites to tomato, there was a $69 \%$ reduction in the number of eggs produced by enzyme-inhibited mites 
Table 4: Effects of acclimation host and assay host on percent of individuals observed feeding in bean-adapted mites (B-population)

\begin{tabular}{|c|c|c|c|c|}
\hline & \multicolumn{2}{|c|}{ Benefit } & \multicolumn{2}{|c|}{ Cost } \\
\hline & $\mathrm{T} \rightarrow \mathrm{T}$ & $\mathrm{B} \rightarrow \mathrm{T}$ & $\mathrm{B} \rightarrow \mathrm{B}$ & $\mathrm{T} \rightarrow \mathrm{B}$ \\
\hline \multicolumn{5}{|l|}{ Observation 1: } \\
\hline Number mites feeding & 20 & 13 & 13 & 15 \\
\hline Number not feeding & 20 & 27 & 27 & 25 \\
\hline Percent feeding & 50 & 33 & 33 & 38 \\
\hline G & \multicolumn{2}{|c|}{2.543} & \multicolumn{2}{|c|}{.220} \\
\hline$P$ & \multicolumn{2}{|c|}{.111} & \multicolumn{2}{|c|}{.639} \\
\hline \multicolumn{5}{|l|}{ Observation 2: } \\
\hline Number mites feeding & 22 & 12 & 8 & 16 \\
\hline Number not feeding & 18 & 28 & 32 & 24 \\
\hline Percent feeding & 55 & 30 & 20 & 40 \\
\hline G & \multicolumn{2}{|c|}{5.176} & \multicolumn{2}{|c|}{3.865} \\
\hline$P$ & \multicolumn{2}{|c|}{.023} & \multicolumn{2}{|c|}{.049} \\
\hline \multicolumn{5}{|l|}{ Observation 3: } \\
\hline Number mites feeding & 20 & 12 & 18 & 17 \\
\hline Number not feeding & 20 & 28 & 22 & 23 \\
\hline Percent feeding & 50 & 30 & 45 & 43 \\
\hline G & \multicolumn{2}{|c|}{3.361} & \multicolumn{2}{|c|}{.051} \\
\hline$P$ & \multicolumn{2}{|c|}{.067} & \multicolumn{2}{|c|}{.822} \\
\hline \multicolumn{5}{|l|}{ Observation 4: } \\
\hline Number mites feeding & 15 & 10 & 14 & 20 \\
\hline Number not feeding & 25 & 30 & 26 & 20 \\
\hline Percent feeding & 38 & 25 & 35 & 50 \\
\hline G & \multicolumn{2}{|c|}{1.462} & \multicolumn{2}{|c|}{1.849} \\
\hline$P$ & \multicolumn{2}{|c|}{.227} & \multicolumn{2}{|c|}{.174} \\
\hline \multicolumn{5}{|l|}{ Overall: } \\
\hline Number mites feeding & 35 & 26 & 30 & 32 \\
\hline Number not feeding & 5 & 14 & 10 & 8 \\
\hline Percent feeding & 88 & 65 & 75 & 80 \\
\hline G & \multicolumn{2}{|c|}{5.77} & \multicolumn{2}{|c|}{.287} \\
\hline$P$ & \multicolumn{2}{|c|}{.016} & \multicolumn{2}{|c|}{.592} \\
\hline
\end{tabular}

Note: Four observations were made, beginning $2 \mathrm{~h}$ after the transfers and repeated every $2 \mathrm{~h} . \mathrm{T} \rightarrow \mathrm{T}$ in observation 1 represents the first sampling of mites acclimated to and assayed on tomato, $\mathrm{B} \rightarrow \mathrm{T}$ in observation 2 represents the second sampling of mites acclimated to bean and assayed on tomato, and so forth. Separate analyses were conducted for the benefits of acclimation and the costs of acclimation. Because the same individuals were observed repeatedly, the $\alpha$ value for the most significant effect has been adjusted using the sequential Bonferroni correction to 0.0125 . An overall analysis is presented last in which organisms were scored for whether they were ever observed feeding in the four samples. Boldface type indicates $P<.05$.

subsequently assayed on tomato compared with controls (mean \pm SE eggs produced by controls, $6.964 \pm 1.061$; inhibited mites, $2.167 \pm 0.555$; treatment, $F=22.29$, df $=$ $1,3, P=.018$; isofemale line, $\mathrm{df}=1, \chi^{2}=2, P=.077$; treatment $\times$ isofemale line, $\mathrm{df}=1, \chi^{2}=0, P=.500$ ).

Feeding mites inhibitors of P-450 detoxification enzymes did not affect the acceptability of tomato (for $3 \mathrm{~h}$ assay, $76 \%$ of inhibited mites and $63 \%$ of control mites accepted tomato, $G=1.265, P=.261$; for $3-\mathrm{d}$ assay, $62 \%$ of inhibited mites and $71 \%$ of control mites accepted tomato, $G=0.629, P=.428$; adjusted $\alpha$ value to reject the null hypothesis $=0.025)$. The effect of inhibiting P-450 enzymes on acceptability of tomato was not variable across isofemale lines (all $P$ 's $\gg .05$ ).

\section{Discussion}

The host range of phytophagous arthropods may be constrained in part by trade-offs in fitness on alternative hosts (Futuyma and Moreno 1988; Jaenike 1990; Karowe 1990; MacKenzie 1996; Traxler and Joern 1999). For both of our laboratory populations of spider mites, mortality was $20 \%$ on bean and twice as high (40\%) on tomato. However, the B-population showed distinctly higher fecundity on bean than on tomato, while the T-population had high fecundity on both hosts. Similar results were shown for mites adapting to bitter cucumber (Gould 1979; Agrawal 2000) and tomato (Fry 1989; Chatzivasileiadis and Sabelis 1997); mites adapted to the novel host do not show reduced fecundity on the original host. Mites adapted to the novel host had high fecundity on both hosts. In these systems, trade-offs were only detectable indirectly by examining "reversion populations" that lost their high performance on the novel host when reverted to the original host for several generations (Gould 1979; Fry 1989; Agrawal 2000). Recently, Yano et al. (2001) have shed light on this mystery by providing evidence that costs of adaptation to novel hosts may take the form of reduced male fitness components, such as mate competition, that are typically not measured. Male mites adapted to tomato were poor competitors for females compared with males adapted to bean (Yano et al. 2001). Thus, poorly competing males in the reversion populations may have contributed to the loss of adaptation to the novel host in former studies. Although genetically based trade-offs in fitness may constrain hostrange expansion, induction of preference and performance may promote the colonization of and adaptation to novel host plants. This induction may contribute to the restricted gene flow observed in local populations of mites and other arthropods (Feder et al. 1994; Tsagkarakou et al. 1997; Agrawal 2001).

Given that mites usually passively disperse on air currents and then can walk to touching hosts, we measured acceptability of hosts, a biologically more relevant measure than strict choice. In fact, dispersal is probably very risky for mites, with few individuals ever finding a new host plant. We found that, when acclimated to a novel host, mites much more readily accepted that host compared with mites that were not acclimated. Nevertheless, mites did not reject their original host if they were acclimated to a novel host. These results were remarkably consistent over 
Table 5: Mixed-model analysis for effects of isofemale line, acclimation host plant, and number of feeding scars on the number of eggs produced on tomato by bean-adapted mites (B-population) using SAS PROC MIXED

\begin{tabular}{lrccr}
\hline Source & \multicolumn{1}{c}{ df } & \multicolumn{1}{c}{$F$} & $\chi^{2}$ & \multicolumn{1}{c}{$P$} \\
\hline Acclimation host $(H)$ & 1,3 & 2.5 & & .212 \\
Feeding scars & 1,62 & 255.52 & & $<.001$ \\
Mite line $(M)^{\text {a }}$ & 1 & & .3 & .292 \\
$M \times H^{\text {a }}$ & 1 & & 0 & .500
\end{tabular}

Note: Boldface type indicates $P<.05$.

${ }^{a}$ Likelihood-ratio $\chi^{2}$ test for random effects.

our 3-d assay period, indicating that acceptance or rejection of the host was a meaningful measure of induced preference. We interpret these results as advantageous to the mites because the mites essentially expand their acceptable host range based on experience. The original high-quality host is not rejected, and the novel host becomes more acceptable. If finding a host is a dangerous activity, as it probably is for many herbivores, then induction of preference may be adaptive irrespective of relative host quality.

Increased performance on novel hosts provides an adaptive hypothesis for the function of induction of preference. Although many studies have shown that herbivores have higher performance on plants on which they were previously reared (e.g., Scriber 1979; Stoyenoff et al. 1994; de Barro et al. 1995), only Karowe (1989) linked the induction of preference with high relative performance. For polyphagous Colius philodice, experience with a particular host plant in the early instars strongly disposed the ultimate instar to prefer that host plant, and those larvae that maintained feeding on their original host plant had higher fecundity than those larvae that were switched (Karowe 1989). Here we also show that acclimation to a novel host plant made that host more acceptable and led to higher fecundity compared with mites that were not acclimated to the novel host. Thus, there are fitness benefits for the plastic response to different host plants. Because phenotypic plasticity is thought to evolve to maximize relative fitness in variable environments (Via and Lande 1985), the benefits and costs of acclimation exemplify adaptive plasticity (sensu Dudley and Schmitt 1996; Agrawal 1998).

In the B-population, the cost of the acclimation response when it was not needed was half of the benefit of the acclimation response when it was needed (fig. 4A). Although our estimate of costs may be considered an upper limit because mites were subject to the other negative effects of acclimation to tomato, two observations suggest that this cost is a realistic measure of detoxification induction. First, tomato-acclimated mites did not show re- duced feeding on bean (table 4). Second, Tetranychus urticae converts food to eggs within a few hours (Helle and Sabelis 1985). Thus, the reduced egg production of tomato-acclimated mites on bean is not likely caused by the lingering effects of tomato. Costs of detoxification induction have not been detectable in other studies (Neal 1987; Appel and Martin 1992), although circumstantial evidence from the parsnip-webworm system suggests that P-450 detoxification is costly (Berenbaum and Zangerl 1994). As with other inducible defense systems (Agrawal 1998; Agrawal and Karban 1999), the costs of detoxification induction in arthropods may relate to energetic requirements or alternative physiological processes such as autotoxicity and hormonal imbalances (Dowd et al. 1983).

When acclimated to the novel host, bean, the Tpopulation also showed increased preference for and performance on the novel host compared with unacclimated mites. However, for this population there appeared to be no cost of acclimation (fig. $5 B$ ). Bean is a highly suitable host and does not appear to require the action of detoxification enzymes (see also Mullin and Croft 1983). We thus tentatively suggest that acclimation to bean by the T-population may be related more to plant nutritional quality than defenses. Alternatively, a nonexclusive hypothesis for the benefit without costs involves detoxification enzymes that are expressed in the mites when on tomato; when the mites are acclimated to bean, detoxification enzyme production may be reduced, and the mites have higher fecundity.

Induction of performance may be related to experiencebased increased feeding on novel hosts. The B-population

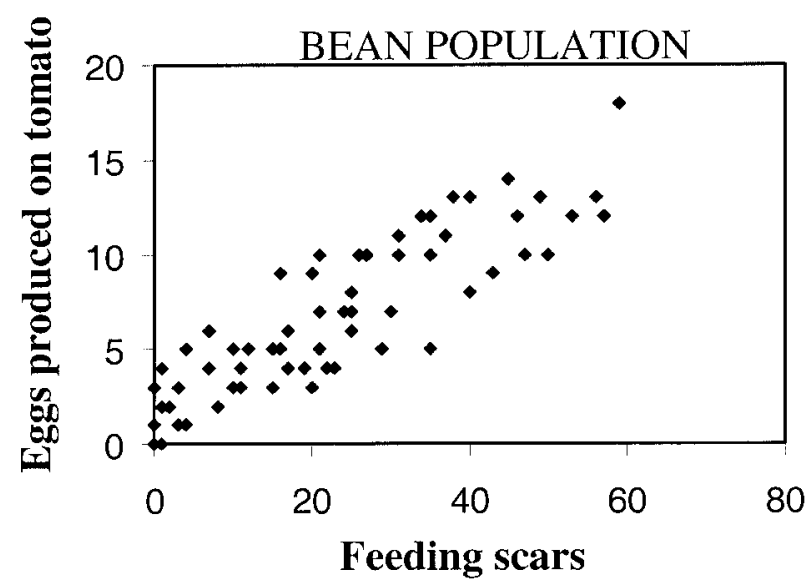

Figure 5: Relationship between number of feeding scars and number of eggs produced for bean-adapted mites (B-population) on tomato. The points include mites acclimated to bean and tomato from the four isofemale lines. 


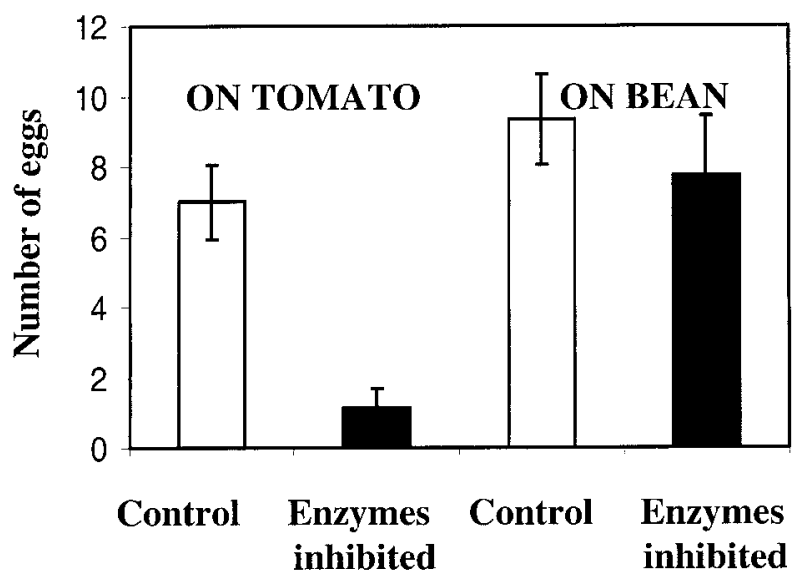

Figure 6: Effects of piperonyl butoxide, an inhibitor of P-450 detoxification enzymes, on performance of bean-adapted mites (B-population) on bean and tomato. Bars are mean \pm SE.

mites acclimated to tomato showed higher feeding and correspondingly higher fecundity on tomato compared with mites that were not acclimated to tomato. However, tomato-acclimated mites (also from the B-population) did not show reduced feeding on bean. This result is consistent with the cost of induction relating to physiological induction of P-450 enzymes and not simply to reduced feeding. It is also consistent with mites not rejecting their original host, irrespective of previous experience. For the B-population of mites, application of piperonyl butoxide (PB), an inhibitor of P-450 detoxification enzymes, strongly reduced mite performance, but only on tomato plants. The P-450 enzymes have been strongly linked to detoxification, and previous physiological studies with $T$. urticae have shown that $\mathrm{PB}$ inhibits this enzymatic activity (Mullin et al. 1984; Strickler and Croft 1985). Thus, we conclude that induction of P-450 enzymes is an important component of induced preference and performance of $T$. urticae on tomato plants.

Increased feeding on acclimated hosts and the induction of detoxification enzymes are not mutually exclusive mechanisms of induction of performance. To the contrary, Spodoptera frugiperda and Manduca sexta larvae showed reduced feeding until detoxification enzymes were induced in their guts (Snyder et al. 1993; Glendinning and Slansky 1995; Snyder and Glendinning 1996). In these studies and ours, induction of performance was correlated with feeding and physiological ability, and the quality of the food per se (i.e., gross growth efficiency; Waldbauer 1968) did not change with induction. In other words, the amount of feeding alone explained the number of eggs produced, without differences in the eggs produced per unit of leaf consumed. For the Colius system, however, induction of preference and performance was correlated with a higher conversion efficiency (Karowe 1989), thus indicating that factors other than induction of detoxification enzymes were probably involved in physiological acclimation. The relationship between detoxification enzymes and behavioral preference remains poorly studied; in our experiments, mites that were fed inhibitors of P-450 enzymes did not show reduced acceptability of the poor host, tomato, which required detoxification.

\section{Acknowledgments}

Thanks to M. Egas, A. Janssen, J. Thaler, A. Weeks, and other members of the Section of Population Biology at the University of Amsterdam for helpful discussions. J. Bruin provided the tomato-adapted mites. The manuscript was improved by comments of E. Bernays, J. Dodgson, M. Egas, R. Hufbauer, R. Karban, N. Kurashige, A. Mooers, K. Rotem, J. Schmitt, J. Thaler, A. Zangerl, and anonymous reviewers. This work was supported by the Section of Population Biology at the University of Amsterdam, U.S. Department of Agriculture NRI Grant 9602065, Natural Sciences and Engineering Research Council of Canada, and the Department of Botany at the University of Toronto.

\section{Literature Cited}

Agrawal, A. A. 1998. Induced responses to herbivory and increased plant performance. Science (Washington, D.C.) 279:1201-1202.

- 2000. Host range evolution: adaptation of mites and trade-offs in fitness on alternate hosts. Ecology 81: 500-508.

-2001. Phenotypic plasticity in the interactions and evolution of species. Science (Washington, D.C.) 294: 321-326.

Agrawal, A. A., and R. Karban. 1999. Why induced defenses may be favored over constitutive strategies in plants. Pages 45-61 in R. Tollrian and C. D. Harvell, eds. The ecology and evolution of inducible defenses. Princeton University Press, Princeton, N.J.

Agrawal, A. A., C. Kobayashi, and J. S. Thaler. 1999. Influence of prey availability and induced host plant resistance on omnivory by western flower thrips. Ecology 80:518-523.

Aina, O. J., J. G. Rodriguez, and D. E. Knavel. 1972. Characterizing resistance to Tetranychus urticae in tomato. Journal of Economic Entomology 65:641-643.

Appel, H. M., and M. M. Martin. 1992. Significance of metabolic load in the evolution of host specificity of Manduca sexta. Ecology 73:216-228.

Berenbaum, M. R., and A. R. Zangerl. 1994. Costs of inducible defense: protein limitation, growth, and detoxification in parsnip webworms. Ecology 75:2311-2317. 
Bernays, E. A., and M. R. Weiss. 1996. Induced food preferences in caterpillars: the need to identify mechanisms. Entomologia Experimentalis et Applicata 78:1-8.

Bolland, H., J. Gutierrez, and C. Flechtmann. 1998. World catalogue of the spider mite family. Brill, Leiden.

Bowers, M. D. 1993. Aposematic caterpillars: lifestyles of the warningly colored and unpalatable. Pages 331-371 in N. E. Stamp and T. M. Casey, eds. Caterpillars: ecological and evolutionary constraints on foraging. Chapman \& Hall, New York.

Brattsten, L. B. 1988. Enzymic adaptations in leaf-feeding insects to host-plant allelochemicals. Iournal of Chemical Ecology 14:1919-1940.

Broadway, R. M. 1995. Are insects resistant to plant proteinase inhibitors? Journal of Insect Phvsiology 41: 107-116.

Byrne, D. H., J. M. Guerrero, A. C. Bellotti, and V. E. Gracen. 1982. Behavior and development of Mononychellus tanajoa (Acari: Tetranychidae) on resistant and susceptible cultivars of cassava. Iournal of Economic Entomology 75:924-927.

Cassida, J. E. 1970. Mixed-function oxidase involvement in the biochemistry of insecticide synergists. ournal of Agricultural and Food Chemistry 18:753-772.

Chatzivasileiadis, E. A., and M. W. Sabelis. 1997. Toxicity of methyl ketones from tomato trichomes to Tetranychus urticae Koch. Experimental and Applied Acarology 21: 473-484.

De Barro, P. J., T. N. Sherratt, O. David, and M. MacLean. 1995. An investigation of the differential performance of clones of the aphid Sitobion avenae on two host species. Oecologia (Berlin) 104:379-385.

Dowd, P., C. M. Smith, and T. C. Sparks. 1983. Detoxification of plant toxins by insects. Insect Biochemistry 13:453-468.

Dudley, S. A., and J. Schmitt. 1996. Testing the adaptive plasticity hypothesis: density-dependent selection on manipulated stem length in Impatiens capensis. American Naturalist 147:445-465.

Dussourd, D. E. 1993. Foraging with finesse caterpillar adaptations for circumventing plant defenses. Pages 92-131 in N. E. Stamp and T. M. Casey, eds. Caterpillars: ecological and evolutionary constraints on foraging. Chapman \& Hall, New York.

- 1997. Plant exudates trigger leaf-trenching by cabbage loopers, Trichoplusia ni (Noctuidae). Oecologia (Berlin) 112:362-369.

Feder, J. L., S. B. Opp, B. Wlazlo, K. Reynolds, W. Go, and S. Spisak. 1994. Host fidelity is an effective premating barrier between sympatric races of the apple maggot fly. Proceedings of the National Academv of Sciences of the USA 91:7990-7994.
Feyereisen, R. 1999. Insect P450 enzymes. Annual Review of Entomology 44:507-533.

Fox, L. R., and P. A. Morrow. 1981. Specialization: species property or local phenomenon? Science (Washington, D.C.) 211:887-893.

Fry, J. D. 1989. Evolutionary adaptation to host plants in a laboratory population of the phytophagous mite $\mathrm{Te}$ tranychus urticae Koch. Oecologia (Berlin) 81:559-565.

- 1990. Trade-offs in fitness on different hosts: evidence from a selection experiment with a phytophagous mite. American Naturalist 136:569-580.

Futuyma, D. J., and G. Moreno. 1988. The evolution of ecological specialization. Annual Review of Ecology and Svstematics 19:207-234.

Glendinning, J. I., and F. Slansky. 1995. Consumption of a toxic food by caterpillars increases with dietary exposure: support for a role of induced detoxification enzymes. Journal of Comparative Physiology A Sensory Neural and Behavioral Physiology 176:337-345.

Gotoh, T., J. Bruin, M. W. Sabelis, and S. B. J. Menken. 1993. Host race formation in Tetranychus urticae: genetic differentiation, host plant preference, and mate choice in a tomato and a cucumber strain. Entomologia Experimentalis et Applicata 68:171-178.

Gould, F. 1979. Rapid host range evolution in a population of the phytophagous mite Tetranychus urticae Koch. Evolution 33:791-802.

Helle, W., and M. W. Sabelis. 1985. Spider mites: their biology, natural enemies, and control. Vol. 1A. Elsevier, Amsterdam.

Jaenike, J. 1990. Host specialization in phytophagous insects. Annual Review of Ecology and Svstematics 21: 243-273.

Karowe, D. 1989. Facultative monophagy as a consequence of prior feeding experience: behavioral and physiological specialization in Colias philodice larvae. Oecologia (Berlin) 78:106-111.

. 1990. Predicting host range evolution: colonization of Coronilla varia by Colias philodice (Lepidoptera: Pieridae). Evolution 44:1637-1647.

Kennedy, G. G., and D. R. Smitley. 1985. Dispersal. Pages 233-242 in W. Helle and M. W. Sabelis, eds. Spider mites: their biology, natural enemies, and control. Vol. 1A. Elsevier, Amsterdam.

Lively, C. M. 1986. Competition, comparative life histories, and maintenance of shell dimorphism in a barnacle. Ecology (Tempe) 67:858-864.

MacKenzie, A. 1996. A trade-off for host plant utilization in the black bean aphid, Aphis fabae. Evolution 50: 155-162.

Mopper, S. 1996. Adaptive genetic structure in phytophagous insect populations. Trends in Ecology \& Evolution 11:235-238. 
Mullin, C. A., and B. A. Croft. 1983. Host-related alterations of detoxification enzymes in Tetranychus urticae (Acari: Tetranychidae). Environmental Entomology 12: 1278-1282.

Mullin, C. A., F. Matsumura, and B. A. Croft. 1984. Epoxide forming and degrading enzymes in the spider mite, Tetranychus urticae. Comparative Biochemistry and Physiology C: Pharmacology Toxicology and Endocrinology 79:85-92.

Neal, J. J. 1987. Metabolic costs of mixed-function oxidase induction in Heliothis zea. Entomologia Experimentalis et Applicata 43:175-180.

Newman, J. A., J. Bergelson, and A. Grafen. 1997. Blocking factors and hypothesis tests in ecology: is your statistics text wrong? Ecology 78:1312-1320.

Papaj, D. R., and R. J. Prokopy. 1989. Ecological and evolutionary aspects of learning in phytophagous insects. Annual Review of Entomology 34:315-350.

Prokopy, R. J., A. L. Averill, S. S. Cooley, and C. A. Roitberg. 1982. Associative learning in egglaying site selection by apple maggot flies. Science (Washington, D.C.) 218:76-77.

Prokopy, R. J., S. S. Cooley, and S. B. Opp. 1989. Prior experience influences the fruit residence of male apple maggot flies, Rhagoletis pomonella. Journal of Insect Behavior 2:39-48.

Rice, W. R. 1989. Analyzing tables of statistical tests. Evolution 43:223-225.

SAS Institute. 1999. SAS/STAT user's guide, release 8 ed. SAS Institute, Cary, N.C.

Scriber, J. M. 1979. The effects of sequentially switching foodplants upon biomass and nitrogen utilization by polyphagous and stenophagous Papilio larvae. Entomologia Experimentalis et Applicata 25:203-215.

Slansky, F., and P. P. Feeny. 1977. Stabilization of the rate of nitrogen accumulation of larvae of the cabbage butterfly on wild and cultivated food plants. Ecological Monographs 47:209-228.

Snyder, M. J., and J. I. Glendinning. 1996. Causal connection between detoxification enzyme activity and consumption of a toxic plant compound. Journal of Comparative Physiology A Sensory Neural and Behavioral Physiology 179:255-261.

Snyder, M. J., E. L. Hsu, and R. Feyereisen. 1993. Induction of cytochrome P-450 activities by nicotine in the tobacco hornworm, Manduca sexta. Journal of Chemical Ecology 19:2903-2916.

Stoyenoff, J. L., J. A. Witter, M. E. Montgomery, and C. A. Chilcote. 1994. Effects of host switching on gypsy moth (Lymantria dispar [L.]) under field conditions. Oecologia (Heidelberg) 97:143-157.

Strickler, K., and B. A. Croft. 1985. Comparative rotenone toxicity in the predator, Amblyseius fallacis (Acari: Phy- toseiidae), and the herbivore, Tetranychus urticae (Acari: Tetranychidae), grown on lima beans and cucumbers. Environmental Entomology 14:243-246.

Szentesi, A., and T. Jermy. 1990. The role of experience in host plant choice by phytophagous insects. Pages 39-74 in E. A. Bernays, ed. Insect-plant interactions. Vol. 2. CRC Press, Boca Raton, Fla.

Tallamy, D. W. 1986. Behavioral adaptations in insects to plant allelochemicals. Pages 273-300 in L. B. Brattsten and S. Ahmad, eds. Molecular aspects of insect-plant associations. Plenum, New York.

Thompson, J. N. 1994. The coevolutionary process. University of Chicago Press, Chicago.

Traxler, M. A., and A. Joern. 1999. Performance trade-offs for two hosts within and between populations of the oligophagous grasshopper Hesperotettix viridis (Acrididae). Oikos 87:239-250.

Tsagkarakou, A., M. Navajas, J. Lagnel, and N. Pasteur. 1997. Population structure in the spider mite Tetranychus urticae (Acari: Tetranychida) from Crete based on multiple allozymes. Heredity 78:84-92.

Van Tienderen, P. H. 1991. Evolution of generalists and specialists in spatially heterogeneous environments. Evolution 45:1317-1331.

Via, S. 1990. Ecological genetics and host adaptation in herbivorous insects: the experimental study of evolution in natural and agricultural systems. Annual Review of Entomology 35:421-446.

. 1991. Specialized host plant performance of pea aphid clones is not altered by experience. Ecology 72: 1420-1427.

Via, S., and R. Lande. 1985. Genotype-environment interaction and the evolution of phenotypic plasticity. Evolution 39:505-522.

Waldbauer, G. P. 1968. The consumption and utilization of food by insects. Advances in Insect Physiology 5: 229-289.

West-Eberhard, M. J. 1989. Phenotypic plasticity and the origins of diversity. Annual Review of Ecology and Systematics 20:249-278.

Yano, S., J. Takabayashi, and A. Takafuji. 2001. Trade-offs in performance on different plants may not restrict the host plant range of the phytophagous mite, Tetranychus urticae. Experimental and Applied Acarology 25: 371-381.

Young, A. M., and M. W. Moffett. 1979. Studies on the population biology of the tropical butterfly Mechanitis isthmia in Costa Rica. American Midland Naturalist 101: 309-319.

Zar, J. H. 1996. Biostatistical analysis. 3d ed. Prentice Hall, Upper Saddle River, N.J.

Associate Editor: Johanna M. Schmitt 